\title{
An open-label, dose-finding study of the combination of satraplatin and gemcitabine in patients with advanced solid tumors
}

\author{
Eugenio Donato Di Paola ${ }^{1,2}$, Silvia Alonso ${ }^{1}$, Rosa Giuliani ${ }^{1}$, Fabio Calabrò ${ }^{1}$, Antonietta D'Alessio ${ }^{1}$, \\ Giovanni Regine ${ }^{3}$, Linda Cerbone ${ }^{1}$, Laura Bianchi ${ }^{1}$, Andrea Mancuso ${ }^{1}$, Sabine Sperka ${ }^{4}$, \\ Marcel Rozencweig ${ }^{5}$ and Cora N. Sternberg ${ }^{1}$ *
}

\author{
' Department of Medical Oncology, San Camillo and Forlanini Hospitals, Rome, Italy \\ 2 Department of Science of Health, School of Medicine, University "Magna Graecia," Catanzaro, Italy \\ ${ }^{3}$ Department of Radiology, San Camillo and Forlanini Hospitals Rome, Italy \\ ${ }^{4}$ Clinical Operations, Agennix AG, Heidelberg, Germany \\ ${ }^{5}$ GPC Biotech Inc., Princeton, NJ, USA
}

\section{Edited by:}

Ganesh V. Raj, University of Texas Southwestern Medical Center, USA

\section{Reviewed by:}

Scott T. Tagawa, Weill Cornell Medical College, USA

Emmanuel S. Antonarakis, Johns Hopkins Sidney Kimmel

Comprehensive Cancer Center, USA

\section{${ }^{*}$ Correspondence:}

Cora N. Sternberg, Department of Medical Oncology, San Camillo and Forlanini Hospitals, Padiglione Flajani, 1st Floor, Circonvallazione

Gianicolense 87, 00152 Rome, Italy. e-mail: cstern@mclink.it
Purpose: Satraplatin is a third generation oral platinum, which has demonstrated antitumor activity. The aim of this phase I study was to determine the maximum tolerated dose (MTD) of the combination of satraplatin and gemcitabine in patients previously treated with chemotherapy and in patients without prior chemotherapy. Patients and Methods: Two separate MTDs were planned in two different patient groups (those with and without prior chemotherapy treatment). Dose escalations were planned in cohorts of three patients. Tumor measurements were obtained every two cycles. Assessment of response was performed according to Response Evaluation Criteria in Solid Tumors (RECIST criteria v.1.0). Results: Thirty subjects were enrolled. A MTD of gemcitabine $1000 \mathrm{mg} / \mathrm{m}^{2}$ days 1 and 8 plus satraplatin $60 \mathrm{mg} / \mathrm{m}^{2}$ days $1-3$, every 21 days was determined in the prior chemotherapy group. No MTD could be determined for the no prior chemotherapy group treated with this schedule. Five patients completed 12 treatment cycles; 22 serious adverse events (SAE) were observed. Although not an entry criteria, overall confirmed response was observed in 17 (24\%) evaluable patients (complete response, CR $=1$ and partial response, $P R=3)$ and in 3/7 (43\%) patients with measure prostate cancer lesions. Conclusions: In this phase lb study, the combination of satraplatin and gemcitabine demonstrated to be safe and efficacious in particular in patients with prostate cancer.

Keywords: satraplatin, oral platinum, phase I study, prostate cancer, solid tumors, chemotherapy

\section{INTRODUCTION}

Satraplatin is a third generation oral platinum complex that has demonstrated activity against several platinum-sensitive and -resistant human tumor cell lines (Twentyman et al., 1992; Kelland et al., 1993; Mellish et al., 1993; Orr et al., 1994; Raynaud et al., 1996).

Preclinical and clinical studies have shown that satraplatin can potentiate the effects of radiotherapy (Van de Vaart et al., 1997; Amorino et al., 1999, 2000; George et al., 2001). In clinical studies, the most frequent dose limiting toxicity (DLT) observed with satraplatin was myelosuppression (leucopenia and thrombocytopenia; McKeage et al., 1995, 1997; Beale et al., 1998; Fokkema et al., 1999; Kurata etal., 2000; George etal., 2001). Of importance, no significant nephrotoxicity or neurotoxicity was reported with satraplatin in either preclinical or clinical studies (McKeage et al., 1993, 1994, 1995, 1997; Beale et al., 1998; Sessa et al., 1998; Fokkema et al., 2000; Vouillamoz-Lorenz et al., 2003; Ricart et al., 2009; Galsky et al., 2012).

In phase II trials, single-agent satraplatin demonstrated activity in patients with small cell lung cancer (SCLC), relapsed ovarian cancer, and prostate cancer (Judson et al., 1997).
In a phase II trial of satraplatin in 39 chemo-naive patients with progressive castration-resistant prostate cancer (CRPC), 7 of $22(32 \%)$ patients had a PSA response, toxicity was mainly hematologic, with grade $3 / 4$ non-hematologic toxicities including transient increases in aspartate transaminase and bilirubin (Latif et al., 2005). These results led the European Organization for Cancer Research (EORTC) to initiate a phase III trial of satraplatin plus prednisone vs prednisone alone for first-line treatment of patients with CRPC (Sternberg et al., 2005). Although the target accrual was 380 patients, only 50 patients were enrolled when the study was terminated early due to a company decision. This trial demonstrated that the combination of satraplatin and prednisone resulted in a significant increase in PSA response compared to prednisone alone (33 vs 9\%; $P=0.046$ ), and improvement in progression-free survival (PFS; 5.2 vs 2.5 months; $P=0.023$; Sternberg et al., 2005).

Encouraging results of this EORTC trial led to development of the SPARC (Satraplatin and Prednisone against Refractory Cancer) study (Sternberg et al., 2009). This trial was a phase III randomized double-blind study in which satraplatin plus prednisone was compared to placebo plus prednisone as second-line 
treatment in patients with CRPC who had received one prior line of cytotoxic chemotherapy. The PFS was 11.1 weeks on the satraplatin and prednisone arm and 9.7 weeks on the placebo and prednisone arm $(P<0.001)$. The median time to pain progression was 66.1 weeks for satraplatin and 22.3 weeks for placebo. A PSA response was observed in 25.4 and $12.4 \%$ of patients, respectively $(P<0.001)$. Despite the improvement in PFS and the palliative effects in favor of satraplatin, overall survival (14.3 months in both arms), the preferred endpoint for regulatory approval by the FDA, for prostate cancer clinical trials as most patients have inevaluable bone disease, was not extended by satraplatin.

Gemcitabine is frequently used in the treatment of several tumor types including: breast, bladder, non-SCLC, and pancreatic cancers. Activity has also been reported in biliary tract, cervical, gall bladder, and ovarian cancers. Gemcitabine has been combined with several different chemotherapeutic agents and has shown activity with platinums, taxanes, anthracyclines, 5-fluorouracil, irinotecan, vinorelbine, and others (Pollera et al., 1994; Glimelius et al., 1996; Burris et al., 1997; Sternberg, 2000).

The rationale for this study was based primarily upon the general synergy between platinum compounds and gemcitabine. Simultaneous drug combination of satraplatin and gemcitabine in UM-UC-3 cells was often more effective than the individual drug treatments but overall showed less than additive effects (GPC Biotech AG, 2005). In this dose finding study the maximum tolerated dose (MTD) of the combination of satraplatin and gemcitabine was evaluated. The hope was to subsequently explore the combination in a variety of solid tumors.

\section{PATIENTS AND METHODS PATIENT SELECTION CRITERIA}

Eligibility criteria included histological diagnosis of metastatic or advanced-stage malignant solid tumors that had progressed following standard therapy or in whom no standard effective treatment was available. Subjects may have received up to two prior lines of chemotherapy for their metastatic disease. Patients with prior therapy with a platinum agent or gemcitabine were allowed as long as they obtained objective response to one of these agents and their relapse occurred after 6 months. Other criteria included: age $\geq 18$ years, ECOG performance status (PS) $0-2$, adequate bone marrow function, adequate renal and hepatic function, measurable or non-measurable disease according to Response Evaluation Criteria in Solid Tumors (RECIST criteria v.1.0).

An independent ethical committee at San Camillo and Forlanini Hospitals approved the protocol. All patients signed written informed consent according to ICH Good Clinical Practice prior to study entry. The trial was conducted according to the Declaration of Helsinki and its amendments.

\section{TRIAL DESIGN AND PROCEDURES}

The study was conducted as a single-center, open label, dose escalation study combining gemcitabine with satraplatin in subjects with advanced solid tumors. Patients were stratified into two groups at registration according to the number of lines of prior chemotherapy (0 vs 1-2). Two separate MTDs were planned for patients with and without previous chemotherapy. The recommended phase II dose was defined as the same dose level as the MTD.

Initially, gemcitabine was given by IV infusion on days 1,8 , and 15 every 28 days followed by satraplatin p.o. for the first 5 days of each cycle (every 28-day schedule, part 1 ). Seventeen patients were enrolled and treated according to this 28 -day schedule. Due to the increasingly common practice of giving gemcitabine and cisplatin on an every 3-week schedule and thrombocytopenia encountered with gemcitabine on the 28-day schedule, the protocol was subsequently amended to an every 21 -day schedule (part 2). The second part of the study included 13 additional patients.

At screening and prior to each gemcitabine infusion, medical history, concomitant medication and PS were recorded. Adverse events were monitored throughout the trial. In both schedules patients were to receive up to 12 cycles. Treatment was discontinued after a dose delay of more than 3 weeks, need for more than one dose reduction, creatinine clearance below $40 \mathrm{ml} / \mathrm{min}$, documented disease progression, or initiation of confounding anti-cancer therapy.

\section{DOSE ESCALATION}

The starting dose and dose escalation of gemcitabine and satraplatin in patients with prior chemotherapy and patients with no prior chemotherapy in parts 1 and 2 of the study are summarized in Tables $\mathbf{1}$ and 2.

Dose escalations were planned in cohorts of three patients. If none of the three patients experienced a DLT during the first cycle, the next three patients were treated at a higher dose level. If any of the three patients experienced a DLT, three additional patients were treated at the same dose. If any of the six patients experienced

Table 1 | Dose escalation in part 1 study (every 28-day schedule).

\begin{tabular}{|c|c|c|c|}
\hline \multirow[t]{2}{*}{ Dose level } & \multirow{2}{*}{$\begin{array}{l}\text { Gemcitabine } \mathrm{mg} / \mathrm{m}^{2} \text { days } \\
1,8 \text {, and } 15^{*}\end{array}$} & \multirow{2}{*}{$\begin{array}{l}\text { Patients with prior chemotherapy } \\
\text { Satraplatin } \mathrm{mg} / \mathrm{m}^{2} / \text { day given daily for } 5 \text { days }\end{array}$} & \multirow{2}{*}{$\begin{array}{l}\text { Patients with no prior chemotherapy } \\
\text { Satraplatin } \mathrm{mg} / \mathrm{m}^{2} / \text { day given daily for } 5 \text { days }\end{array}$} \\
\hline & & & \\
\hline+1 & 1000 & 40 & 60 \\
\hline+2 & 1000 & 60 & 80 \\
\hline
\end{tabular}

${ }^{*}$ Cycles are to be repeated every 28 days. 
Table 2 | Dose escalation in part 2 study (every 21-day schedule).

\begin{tabular}{lllll}
\hline Dose level & $\begin{array}{l}\text { Gemcitabine } \mathbf{m g} / \mathbf{m}^{\mathbf{2}} \text { days } \\
\mathbf{1} \text { and } \mathbf{8}^{*}\end{array}$ & Patients with prior chemotherapy & & Patients with no prior chemotherapy \\
& & Satraplatin $\mathbf{~} \mathbf{g} / \mathbf{m}^{\mathbf{2}} / \mathbf{d a y}$ given daily for $\mathbf{3}$ days & Satraplatin $\mathbf{~} \mathbf{g} / \mathbf{m}^{\mathbf{2}} / \mathbf{d a y}$ given daily for $\mathbf{3}$ days \\
\hline Starting & 1000 & 60 & 80 \\
+1 & 1000 & 80 & 100 & 120 \\
+2 & 1000 & 100 & 120 \\
+3 & 1250 & &
\end{tabular}

${ }^{*}$ Cycles are to be repeated every 21 days.

a DLT, the MTD was exceeded and three additional patients had to be treated at the lower dose (if only three patients were previously treated at that dose). The MTD was defined as the dose level at which $0 / 6$ or $1 / 6$ patients experience DLTs with the next higher dose having at least $2 / 3$ or $2 / 6$ patients experiencing a DLT.

Toxicities were graded on the basis of Common Terminology Criteria for Adverse Events (CTCAE) version 3.0. A DLT was defined as a drug-related CTCAE G3 or G4 non-hematologic toxicity (except reversible emesis or diarrhea) or G4 neutropenia of more than 7 days and/or complicated by infection or G3G4 thrombocytopenia or any bleeding episode requiring platelet transfusion, or delayed recovery (to G1 or baseline, except alopecia). A toxicity related to the combination treatment which delayed initiation of the next cycle by more than 2 weeks was also a DLT.

\section{SAFETY ASSESSMENT}

Safety was assessed weekly by physical examination, vital signs and laboratory measurements. Subjects were followed for adverse events for at least 30 days after the last dose of therapy. All studyrelated adverse events were followed to resolution or stabilization.

\section{TUMOR ASSESSMENTS}

Subjects with measurable disease who had completed at least two cycles of study treatment and had at least one disease assessment following the initiation of study treatment were considered evaluable for disease response. Tumor measurements were obtained every two cycles of treatment. Response was assessed according to RECIST criteria v.1.0 (Therasse et al., 2000). Subjects who discontinued for toxicity or after completion of 12 cycles without disease progression were followed every 3 months up to 1 year or until progressive disease, subsequent anti-tumor treatment, or death.

\section{STATISTICAL METHODS}

The statistical analysis was only descriptive.

\section{RESULTS}

\section{PATIENT CHARACTERISTICS}

Thirty patients were entered onto the study. Of these, 17 were in part 1 (every 28-day schedule) and 13 in part 2 (every 21-day schedule). Patient characteristics of both groups are described in Table 3.

\section{TREATMENT, MTD, AND DLTs}

The median number of cycles administered was 3. Five patients completed a total of 12 treatment courses, the maximum number of cycles per protocol. Details are reported in Table 4.

\section{Every 28-day schedule part I of the trial}

The trial was initiated with a 28 -day schedule. The starting dose of satraplatin was $40 \mathrm{mg} / \mathrm{m}^{2}$ in pretreated and $60 \mathrm{mg} / \mathrm{m}^{2}$ in chemonaive patients (equivalent to 40 and $60 \%$ of the MTD of singleagent satraplatin).

In the "previous chemotherapy" group, three patients were treated at the starting dose (gemcitabine $800 \mathrm{mg} / \mathrm{m}^{2}$ days 1,8 , and 15 plus satraplatin $40 \mathrm{mg} / \mathrm{m}^{2}$ days $1-5$; every 28 days). A DLT (G3 transaminases) was observed and the cohort was expanded to three more patients. Another DLT (G3 transaminases) was observed in this cohort. Thus, no patients were treated at the next dose level. Since two DLTs occurred, no MTD could be determined.

In the "no previous chemotherapy" group, eight patients were treated at the starting dose (gemcitabine $800 \mathrm{mg} / \mathrm{m}^{2}$ days 1,8 , and 15 plus satraplatin $60 \mathrm{mg} / \mathrm{m}^{2}$ days $1-5$; every 28 days). Two patients were not evaluable for the MTD and were replaced. Both patients did not receive treatment on days 8 and 15 of cycle 1 , due to G3 thrombocytopenia or withdrawal of consent during cycle 1. One DLT (G3 diarrhea) was observed in one of the six evaluable patients. Therefore, three additional patients received +1 dose level (gemcitabine $1000 \mathrm{mg} / \mathrm{m}^{2}$ days 1 , 8, and 15 plus satraplatin $60 \mathrm{mg} / \mathrm{m}^{2}$ days $1-5$; every 28 days). At dose level +1 , one DLT (G3 thrombocytopenia) was observed in one of three patients. The starting dose (level 0 ) was therefore determined as the MTD.

In the "previous chemotherapy" group, no MTD was determined, and in the "no previous chemotherapy" group, the MTD was determined at dose level 0 . Only 6 of 17 patients received gemcitabine on D8 and D15. Therefore, the protocol was amended in both groups to a more convenient every 3 -week schedule.

\section{Every 21-day schedule part II of the trial}

In the "previous chemotherapy" group, seven patients were treated at the starting dose (gemcitabine $1000 \mathrm{mg} / \mathrm{m}^{2}$ days 1 and 8 plus satraplatin $60 \mathrm{mg} / \mathrm{m}^{2}$ days $1-3$; every 21 days). One patient was not evaluable for the MTD, as he received no treatment on day 8 due to urinary infection. One DLT (G3 thrombocytopenia) was observed in one of six evaluable patients. 
Table 3 | Patients characteristic.

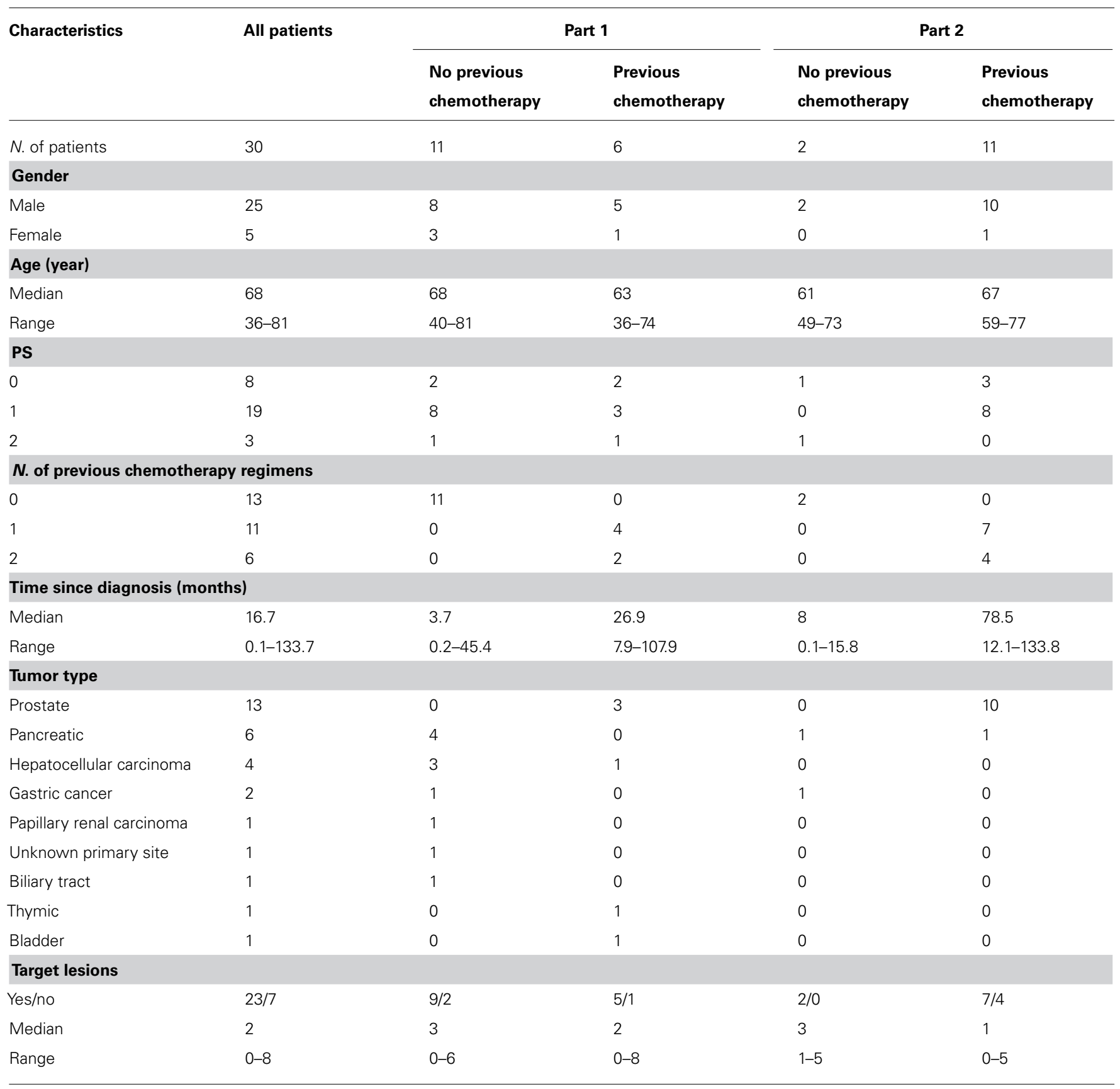

In the next three patients, the dose was escalated to dose level +1 (gemcitabine $1000 \mathrm{mg} / \mathrm{m}^{2}$ days 1 and 8 plus satraplatin $80 \mathrm{mg} / \mathrm{m}^{2}$ days $1-3$; every 21 days). Two DLTs were observed (G3 thrombocytopenia).

The starting dose (level 0) was, therefore, determined as the MTD and further enrolment of six patients was planned. This was stopped after the first patient exhibited a DLT (G3 thrombocytopenia) due to a decision of the sponsor.

In the "no previous chemotherapy" group, two patients were treated at the starting dose (gemcitabine $1000 \mathrm{mg} / \mathrm{m}^{2}$ days 1 and 8 plus satraplatin $80 \mathrm{mg} / \mathrm{m}^{2}$ days $1-3$; every 21 days). In each patient, one DLT was observed (G3 neutropenia and G3 thrombocytopenia). Dose level 0 was closed and the MTD could not be determined.

\section{Safety results}

All 30 patients received at least one dose of study treatment. A total of 137 cycles were evaluated for safety and 310 non-serious adverse drug reactions (ADRs) were recorded. Eighteen patients experienced 30 SAEs including 22 that were considered "possibly related" to study treatment. The reported serious ADRs were: diarrhea (1), rectal hemorrhage (1), ALT increase (3), low hemoglobin (4), low 
Table 4 | Treatment (cycles administered).

\begin{tabular}{|c|c|c|c|c|c|}
\hline \multirow{2}{*}{$\begin{array}{l}N . \text { of cycles } \\
\text { administered }\end{array}$} & \multirow[t]{2}{*}{ All patients } & \multicolumn{2}{|c|}{ Part 1} & \multicolumn{2}{|c|}{ Part 2} \\
\hline & & $\begin{array}{l}\text { No previous } \\
\text { chemotherapy }\end{array}$ & $\begin{array}{l}\text { Previous } \\
\text { chemotherapy }\end{array}$ & $\begin{array}{l}\text { No previous } \\
\text { chemotherapy }\end{array}$ & $\begin{array}{l}\text { Previous } \\
\text { chemotherapy }\end{array}$ \\
\hline Total & 137 & 38 & 25 & 4 & 70 \\
\hline Median & 3 & 2 & 2 & 2 & 6 \\
\hline 12 cycles & 5 & 1 & 1 & 0 & 3 \\
\hline
\end{tabular}

platelets (8), neutropenia (2), elevated bilirubin (1), and deep venous thrombosis (1). G4 toxicities occurred in seven patients (three neutropenia, two anemia, one thrombocytopenia, and one pain). The predominant adverse event that occurred in greater than $20 \%$ of the patients are reported in Table 5. No major differences in adverse events G3-G4 were observed between the subjects in the two groups with and without prior chemotherapy.

\section{ANTITUMOR ACTIVITY}

Seventeen of the 30 patients were evaluable for objective tumor response. Thirteen patients were excluded from the analysis as per protocol because seven had no measurable disease (only nontarget lesions) at inclusion, four received less than two cycles of study treatment and two patients had no further disease assessments following initiation of the study treatment.

One patient with pancreatic cancer and one target lesion in the liver showed a complete response (CR) at cycle 2 which was confirmed at cycle 4 . Time to progression was 422 days. Three patients had confirmed partial response (PR). Seven had stable disease (SD) in whom two had a PR that was not confirmed. Six patients had progression disease (PD). All three patients who obtained a PR had metastatic CRPC. Two patients had target lesions in lymph nodes and in one the liver. Of the two patients that had an unconfirmed PR, one had metastatic CRPC with target lesions in lymph nodes and the other had a biliary tract tumor with four target lesions (two in the liver and two in lymph nodes). The overall response rate $(\mathrm{RR} ; \mathrm{CR}+\mathrm{PR})$ in all $(4 / 17)$ evaluable patients was $24 \%$.

\section{DISCUSSION}

The purpose of this study was to determine the MTD and DLT of gemcitabine in combination with satraplatin in two groups of patients with advanced solid tumors. Since there was strong evidence of synergism of the combination of the two drugs in preclinical studies, it was decided to conduct the study in two groups of patients simultaneously.

The first group included patients previously treated with one or two lines of chemotherapy and for whom no further standard treatment was available. The second group included patients not previously treated with chemotherapy and in whom there was no standard treatment. This group included patients with pancreatic, gastric, hepatocellular carcinoma, biliary tract, or papillary renal cell cancers (Table 3).According to the protocol, more patients on the 28-day schedule at dose level 0 should have been included in order to verify if this was the recommended dose for phase II trials. However, taking into account the encouraging evidence of activity of the combination of the two drugs, as evidenced by a CR in a patient with pancreatic cancer, one PR in a patient with CRPC and one unconfirmed PR in a patient with biliary tract cancer, and the

Table 5 | Adverse events in $\geq \mathbf{2 0} \%$ of patients.

\begin{tabular}{|c|c|c|c|c|c|}
\hline Adverse events & $\begin{array}{l}N . \text { of patients } \\
\text { with G1 }\end{array}$ & $\begin{array}{l}\text { N. of patents } \\
\text { with } \mathbf{G} 2\end{array}$ & $\begin{array}{l}N . \text { of patents } \\
\text { with G3 }\end{array}$ & $\begin{array}{l}\text { N. of patents } \\
\text { with } \mathbf{G} 4\end{array}$ & $\begin{array}{l}\% \text { of patients } \\
\text { with (G1-G4) }\end{array}$ \\
\hline Asthenia & 6 & 12 & 4 & 0 & 73 \\
\hline Nausea & 8 & 7 & 1 & 0 & 53 \\
\hline Thrombocytopenia & 3 & 4 & 7 & 1 & 50 \\
\hline Neutropenia & 1 & 3 & 7 & 3 & 47 \\
\hline Anorexia & 5 & 8 & 1 & 0 & 47 \\
\hline Anemia & 0 & 8 & 2 & 2 & 40 \\
\hline Fever & 7 & 4 & 0 & 0 & 37 \\
\hline Diarrhea & 3 & 4 & 2 & 0 & 30 \\
\hline Constipation & 9 & 0 & 0 & 0 & 30 \\
\hline Pain & 5 & 1 & 0 & 1 & 23 \\
\hline Edema & 5 & 1 & 0 & 0 & 20 \\
\hline
\end{tabular}


fact that only 6 of 17 patients had received gemcitabine on days 8 and 15, the protocol was amended in both groups to a more feasible schedule (gemcitabine days 1 and 8, plus satraplatin days 1-3; every 21 days).

Unfortunately, the second part of the amended study was stopped after the FDA ODAC meeting in which satraplatin and prednisone in the SPARC trial failed to reveal an OS advantage in patients with CRPC and one prior line of chemotherapy. At that time only $50 \%$ of patients in the SPARC trial had received prior first-line treatment with docetaxel which was becoming the standard first-line chemotherapy.

The main objective of the study was to explore the safety of the combination of gemcitabine with satraplatin. For this reason, measurable disease was not required in the study. Of particular interest was the activity in patients with metastatic CRPC. The cycle length of 21 days would have provided a practical schedule. In addition, the efficacy of platinums in prostate cancer has been noted. An oral platinum compound would have potentially been a very efficacious alternative to other chemotherapeutic agents $(\mathrm{Oh}$ et al., 2007). There were 13 CRPC patients enrolled, all of them were included after treatment with docetaxel and prednisone, and four also received a prior second line of chemotherapy.

Only seven CRPC patients had target lesions and were evaluable according to the protocol, while six were not evaluable for objective response. Four were not evaluable due to lack of measurable target lesions, one received less than two cycles and one was not re-evaluated. In this subgroup of evaluable patients with measurable lesions, the objective RR was $43 \%$. Although the number of evaluable CRPC patients was limited, the data are reinforced by the fact that PSA responses $>50 \%$ according to Prostate Specific Antigen Working Group (PSAWG) criteria were also observed in $4 / 12(33 \%)$ patients.

\section{REFERENCES}

Amorino, G. P., Freeman, M. L., Carbone, D., Lebwohl, D. E., and Choy, H. (1999). Radiopotentiation by the oral platinum agent, JM216: role of repair inhibition. Int. J. Radiat. Oncol. Biol. Phys. 44, 399-405.

Amorino, G. P., Mohr, P. J., Hercules, S. K., Pyo, H., Choy, H., et al. (2000). Combined effects of the orally active cisplatin analog, JM216, and radiation in antitumor therapy. Cancer Chemother. Pharmacol. 46, 423-426.

Beale, P., Raynaud, F., Hanwell, J., Berry, C., Moore, S., Odell, D., et al. (1998). Phase I study of oral JM216 given twice daily. Cancer Chemother. Pharmacol. 42, 142-148.

Burris, H. A. III, Moore, M. J., Andersen, J., Green, M. R., Rothenberg, M. L., Modiano, M. R., et al. (1997). Improvements in survival and clinical benefit with gemcitabine as first-line therapy for patients with advanced pancreas cancer: a randomized trial. J. Clin. Oncol. 15, 2403-2413.

Fokkema, E., de Vries, E. G., Meijer, S., and Groen, H. J. (2000). Lack of

It is unfortunate that development of satraplatin was halted as it showed potentially interesting results in this study and other studies in ovarian cancer, lung cancer and as a radioenhancer for external beam radiation therapy. In actuality, satraplatin is under development as a radioenhancer and translational research studies with biomarkers are supported through a Material Cooperative Research and Development Agreement (MCRADA) with the NIH.

\section{CONCLUSION}

The arsenal of novel hormonal treatment for patients with CRPC has radically changed in recent years. Nonetheless, the results of this trial showed that the combination of gemcitabine and satraplatin, in particular given in an every 3 week cycle is feasible and has potential anti-tumor activity.

The results were encouraging, in particular, as second and third line chemotherapy in patients with CRPC, supported by the high objective RR of $43 \%$. While the drug combination may be of interest in CRPC, the study did not prospectively enrolled a defined cohort of CRPC patients. Although the number of evaluable patients was limited due to study closure, the combination was feasible and patients were able to receive the maximum number of cycles permitted by the protocol ( 12 cycles).

In conclusion, the results of this study and the compliance with an oral platinum agent support the further development of satraplatin to better quantify its activity and safety in patients with CRPC after receiving docetaxel and other novel therapies such as abiraterone or enzalutamide. The combination was also interesting in pancreatic and biliary tumors.

\section{ACKNOWLEDGMENT}

This study was supported by GPC Biotech Inc., Princeton, NJ, USA.

(1996). Chemotherapy improves survival and quality of life in advanced pancreatic and biliary cancer. Ann. Oncol. 7, 593-600.

GPC Biotech AG. (2005). Combination Treatment of JM118 and Gemcitabine is Synergistic in Human Bladder Carcinoma Cells (UM-UC-3) Study Report. Martinsried: GPC Biotech AG.

Judson, I., Cerny, T., Epelbaum, R., Dunlop, D., Smyth, J., Schaefer, B., et al. (1997). Phase II trial of the oral platinum complex JM216 in non small-cell lung cancer: an EORTC early clinical studies group investigation. Ann. Oncol. 8, 604-606.

Kelland, L. R., Abel, G., McKeage, M. J., Jones, M., Goddard, P. M., Valenti, M., et al. (1993). Preclinical antitumor evaluation of bis-acetatoammine-dichloro-cyclohexylamine platinum(IV): an orally active platinum drug. Cancer Res. 53, 2581-2586.

Kurata, T., Tamura, T., Sasaki, Y. Fujii, H., Negoro, S., and Fukuoka, M. (2000). Pharmacokinetic and pharmacodynamic analysis of bis-acetato-ammine-dichloro-cyclohexylamine-platinum(IV) (JM216) administered once a day for five consecutive days: a phase I study. Jpn. J. Clin. Oncol. 30, 377-384.

Latif, T., Wood, L., Connel, C., Smith, D. C., Vaughn, D., Lebwohl, D., et al. (2005). Phase II study of oral bis (aceto) ammine dichloro (cyclohexamine) platinum (IV) (JM-216, BMS-182751) given daily $x 5$ in hormone refractory prostate cancer (HRPC). Invest. New Drugs 23, 79-84. McKeage, M. J., Boxall, F., Jones, M., and Harrap, K. R. (1994). Lack of neurotoxicity of oral bisacetatoamminedichlorocyclohexylamine-platinum(IV) in comparison to cisplatin and tetraplatin in the rat. Cancer Res. 54, 629-631.

McKeage, M. J., Mistry, P., Ward, J., Boxall, F. E., Loh, S., O’Neill, C., etal. (1995). A phase I and pharmacology study of an oral platinum complex, JM216: dose-dependent pharmacokinetics with single-dose administration. Cancer Chemother. Pharmacol. 36, 451-458. 
McKeage, M. J., Morgan, S. E., Boxall, F., Murrer, B. A., Hard, G. C., and Harrap, K. R. (1993). Lack of nephrotoxicity of oral ammine/amine platinum (IV) dicarboxylate complexes in rodents. Br. J. Cancer 67, 9961000.

McKeage, M. J., Raynaud, F., Ward, J., Berry, C., O’Dell, D., Kelland, L. R., et al. (1997). Phase I and pharmacokinetic study of an oral platinum complex given daily for 5 days in patients with cancer. J. Clin. Oncol. 15, 2691-2700.

Mellish, K. J., Kelland, L. R., and Harrap, K. R. (1993). In vitro platinum drug chemosensitivity of human cervical squamous cell carcinoma cell lines with intrinsic and acquired resistance to cisplatin. Br. J. Cancer 68, 240-250.

Oh, W. K., Tay, M. H., and Huang, J. (2007). Is there a role for platinum chemotherapy in the treatment of patients with hormone-refractory prostate cancer? Cancer 109, 477-486.

Orr, R. M., O'Neill, C., Nicolson, M., Barnard, C. F., Murrer, B. A., Giandomenico, C. M., etal. (1994). Evaluation of novel ammine/amine platinum (IV) dicarboxylates in L1210 murine leukaemia cells sensitive and resistant to cisplatin, tetraplatin or carboplatin. $\mathrm{Br}$. J. Cancer 70, 415-420.
Pollera, C. F., Ceribelli, A., Crecco, M., and Calabresi, F. (1994). Weekly gemcitabine in advanced bladder cancer: a preliminary report from a phase I study. Ann. Oncol. 5, 182-184.

Raynaud, F. I., Mistry, P., Donaghue, A., Poon, G. K., Kelland, L. R., Barnard, C. F., et al. (1996). Biotransformation of the platinum drug JM216 following oral administration to cancer patients. Cancer Chemother. Pharmacol. 38, 155-162.

Ricart, A. D., Sarantopoulos, J., Calvo, E., Chu, Q. S., Greene, D., Nathan, F. E., et al. (2009). Satraplatin, an oral platinum, administered on a five-day every-five-week schedule: a pharmacokinetic and food effect study. Clin. Cancer Res. 15, 3866-3871.

Sessa, C., Minoia, C., Ronchi, A., Zucchetti, M., Bauer, J., Borner, M., et al. (1998). Phase I clinical and pharmacokinetic study of the oral platinum analogue JM216 given daily for 14 days. Ann. Oncol. 9, 1315-1322.

Sternberg, C. N. (2000). Gemcitabine in bladder cancer. Semin. Oncol. 27, 31-39.

Sternberg, C. N., Petrylak, D. P., Sartor, O., Witjes, J. A., Demkow, T., Ferrero, J. M., et al. (2009). Multinational, double-blind, phase III study of prednisone and either satraplatin or placebo in patients with castraterefractory prostate cancer progressing after prior chemotherapy: the
SPARC trial. J. Clin. Oncol. 27, 54315438.

Sternberg, C. N., Whelan, P., Hetherington, J., Paluchowska, B., Slee, P. H., Vekemans, K., et al. (2005). Phase III trial of satraplatin, an oral platinum plus prednisone vs. prednisone alone in patients with hormone-refractory prostate cancer. Oncology 68, 2-9.

Therasse, P., Arbuck, S. G., Eisenhauer, E. A., Wanders, J., Kaplan, R. S. Rubinstein, L., et al. (2000). New guidelines to evaluate the response to treatment in solid tumors (RECIST guidelines). J. Natl. Cancer Inst. 92, 205-216.

Twentyman, P. R., Wright, K., Mistry, P., Kelland, L. R., and Murrer, B. A. (1992). Sensitivity to novel platinum compounds of panels of human lung cancer cell lines with acquired and inherent resistance to cisplatin. Cancer Res. 52, 5674-5680.

Van de Vaart, P., Klaren, H. M., Hofland, I., and Begg, A. C. (1997). Oral platinum analogue JM216, a radiosensitizer in oxic murine cells. Int. J. Radiat. Biol. 72, 675-683.

Vouillamoz-Lorenz, S., Buclin, T., Lejeune, F., Bauer, J., Leyvraz, S., and Decosterd, L. A. (2003). Pharmacokinetics of satraplatin (JM216), an oral platinum (IV) complex under daily oral administration for 5 or 14 days. Anticancer Res. 23, $2757-$ 2765.
Conflict of Interest Statement: Marcel Rozencweig was consultant for and Sabine Sperka was employed at GPC Biotech Inc. The other authors declare that the research was conducted in the absence of any commercial or financial relationships that could be construed as a potential conflict of interest.

Received: 17 August 2012; paper pending published: 12 September 2012; accepted: 05 November 2012; published online: 22 November 2012.

Citation: Donato Di Paola E, Alonso $S$, Giuliani R, Calabrò F, D’Alessio A, Regine G, Cerbone L, Bianchi L, Mancuso A, Sperka S, Rozencweig $M$ and Sternberg CN (2012) An open-label, dosefinding study of the combination of satraplatin and gemcitabine in patients with advanced solid tumors. Front. Oncol. 2:175. doi: 10.3389/fonc.2012.00175

This article was submitted to Frontiers in Genitourinary Oncology, a specialty of Frontiers in Oncology.

Copyright () 2012 Donato Di Paola, Alonso, Giuliani, Calabrò, D'Alessio, Regine, Cerbone, Bianchi, Mancuso, Sperka, Rozencweig and Sternberg. This is an openaccess article distributed under the terms of the Creative Commons Attribution License, which permits use, distribution and reproduction in other forums, provided the original authors and source are credited and subject to any copyright notices concerning any third-party graphics etc. 